\title{
Correlated Electron Systems of Different Dimensionalities
}

\author{
J. KURZYK, W. WÓJCIK \\ Instytut Fizyki, Politechnika Krakowska \\ Podchorążych 1, 30-084 Kraków, Poland
}

AND J. SPAŁEK

Instytut Fizyki im. Mariana Smoluchowskiego, Uniwersytet Jagielloński

Reymonta 4, 30-059 Kraków, Poland

The optimized single-particle wave functions contained in the parameters of the Hubbard model (hopping integral $t$ and intraatomic interaction $U)$ are determined explicitly in the correlated state for electronic systems of various symmetries and dimensions: Hubbard chain, square and triangular lattices, and the three cubic lattices: SC, BCC, and FCC. In effect, the electronic properties of these structures as a function of the interatomic distance $R$ are obtained. In most cases, the model parameters do not scale linearly with the lattice constant. Also, the atomic part of the total ground state energy changes with the $U / t$ ratio and therefore should be (and is) included in the analysis. The solutions of dimensions $D>1$ are analyzed by utilizing the approximate Gutzwiller treatment.

PACS numbers: 71.27.+a, 71.30.+h, 71.10.Fd

\section{Introduction}

We extended the method (exact diagonalization combined with the ab initio approach, EDABI) used earlier for analysis of correlated nanoscopic systems [1-5] to infinite (periodic) $s$-band-like systems described by the Hubbard model and its extensions. We describe correlated electron systems of different dimensionalities and symmetries by using the extended Hubbard Hamiltonian

$$
H=\varepsilon_{a}^{\mathrm{eff}}+\sum_{\langle i j\rangle \sigma} t_{i j} c_{i \sigma}^{\dagger} c_{j \sigma}+U \sum_{i} n_{i \uparrow} n_{i \downarrow},
$$

where $t_{i j}$ - the hopping integral between the sites $i$ and $j, U$ is the magnitude of the Coulomb interaction at given site $i$, and 


$$
\varepsilon_{a}^{\mathrm{eff}} \stackrel{\text { a.u. }}{=} \varepsilon_{a}+\frac{1}{N} \sum_{i<j}\left(K_{i j}+\frac{2}{R_{i j}}\right)
$$

is the effective atomic energy reducing to the true atomic energy $\varepsilon_{a}$ in the limit of large interatomic separation $\left(K_{i j}\right.$ is the corresponding quantity for electrons located on sites $i$ and $j \neq i$ ). This energy is important in view of the fact that we will study the system evolution as a function of the interatomic distance $R$. In that situation $\varepsilon_{a}^{\text {eff }}$ is not a constant quality, as would be in the case for a parametrized model, but reduces to the atomic energy of the hydrogenic atom in the $R \rightarrow \infty$ limit.

In order to determine the ground state energy we use the Gutzwiller ansatz [6] (see also [7] where we used the exact Lieb-Wu solution as well as the Gutzwiller wave function approximation (GA) for the Hubbard chain)

$$
\frac{E_{\mathrm{G}}}{N} \equiv \varepsilon_{a}^{\mathrm{eff}}-|\bar{\varepsilon}|\left(1-U / U_{\mathrm{c}}\right)^{2}, \quad U_{\mathrm{c}}=8|\bar{\varepsilon}|,
$$

where $\bar{\varepsilon}$ is the average kinetic energy of noninteracting electrons.

The formula represents a final step in solving model in GA scheme. Here, it represents a starting point in obtaining the optimized single-particle wave functions $\left\{w_{i}(\boldsymbol{r})\right\}$. We construct the single-particle wave functions $\left\{w_{i}(\boldsymbol{r})\right\}$ entering the expressions for $t, U, \varepsilon_{a}^{\mathrm{eff}}$, and $K_{i j}$ in the following manner:

$$
w_{i}(\boldsymbol{r}) \equiv \beta \Psi_{i}(\boldsymbol{r})-\gamma \sum_{j=1}^{z} \Psi_{j}(\boldsymbol{r}),
$$

where $z$ is the number of nearest neighbors, $\beta$ and $\gamma$ are the mixing coefficients, and

$$
\Psi_{i}(\boldsymbol{r}) \equiv \sqrt{\frac{\alpha^{3}}{\pi}} \mathrm{e}^{-\alpha\left|\boldsymbol{r}-\boldsymbol{R}_{i}\right|}
$$

is the $1 s$-like atomic wave function centered around the site $i$. In concrete calculations they are represented by the Gaussians from the STO-3G basis. The parameters $\beta$ and $\gamma$ are selected to fulfill the orthogonality of the basis. We optimized the single-particle wave function by changing the $\alpha$ parameter in order to achieve the global minimum of the ground state energy for all interatomic distances under consideration.

\section{Results and discussion}

In Fig. 1 we display the ground state energy as a function of interatomic distance for Hubbard chain $(\mathrm{CH})$, square (SQ) and triangular (TR) lattices. We see the $U / t$ ratio similar for $\mathrm{CH}$ and $\mathrm{SQ}$ (cf. inset). Figure 2 shows the ground state energy and the $U / t$ ratio as a function of interatomic distance for cubic lattices. In Fig. 3 we display the band $\left(-E_{\mathrm{B}}=-8 d(1-2 d)|\bar{\varepsilon}|\right)$ and interaction $\left(E_{\text {int }}=U d\right)$ energies near critical interatomic distance for all lattices under consideration. One can see that $-E_{\mathrm{B}} \cong E_{\text {int }}$ near critical interatomic distance. Moreover, we see that the metal-insulator (Mott) transition (MIT) for a square lattice is located almost 


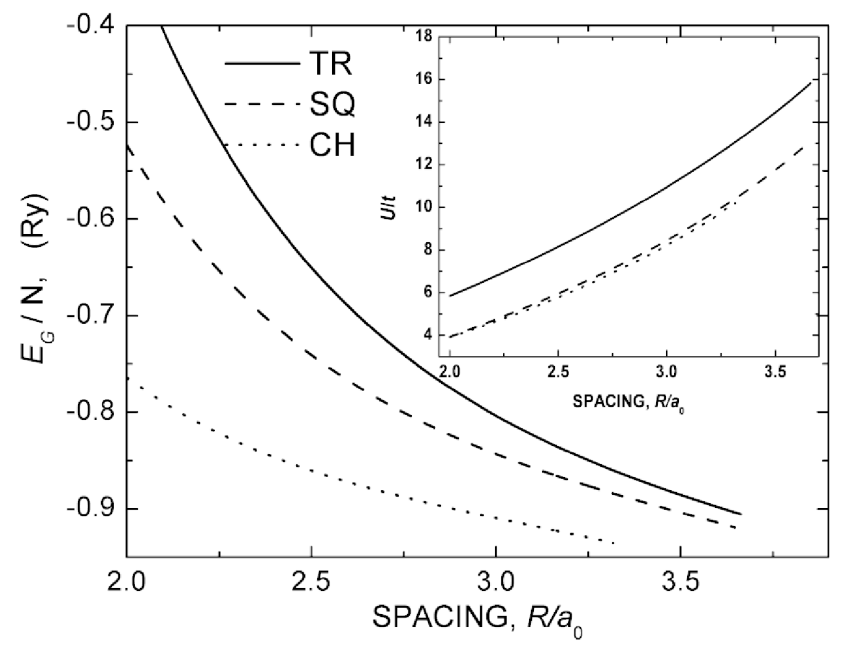

Fig. 1. The ground state energy vs. $R$ for $\mathrm{CH}, \mathrm{SQ}$, and TR lattices for the Gutzwiller ansatz. Inset: $U / t$ ratio as a function of lattice parameter.

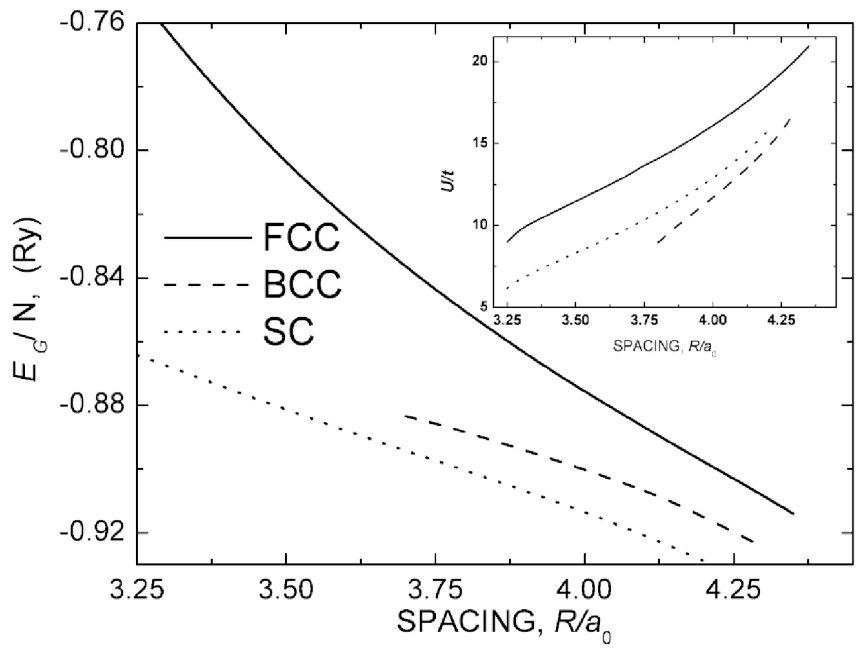

Fig. 2. The ground state energy vs. $R$ for SC, BCC and FCC lattices for the Gutzwiller ansatz. Inset: $U / t$ ratio as a function of lattice parameter.

in the same point as that for a triangular lattice. Cubic lattices have also very similar critical distances.

In summary, we determined mean-field (Gutzwiller ansatz) MIT boundaries for lattices of different dimensionality and symmetry. 


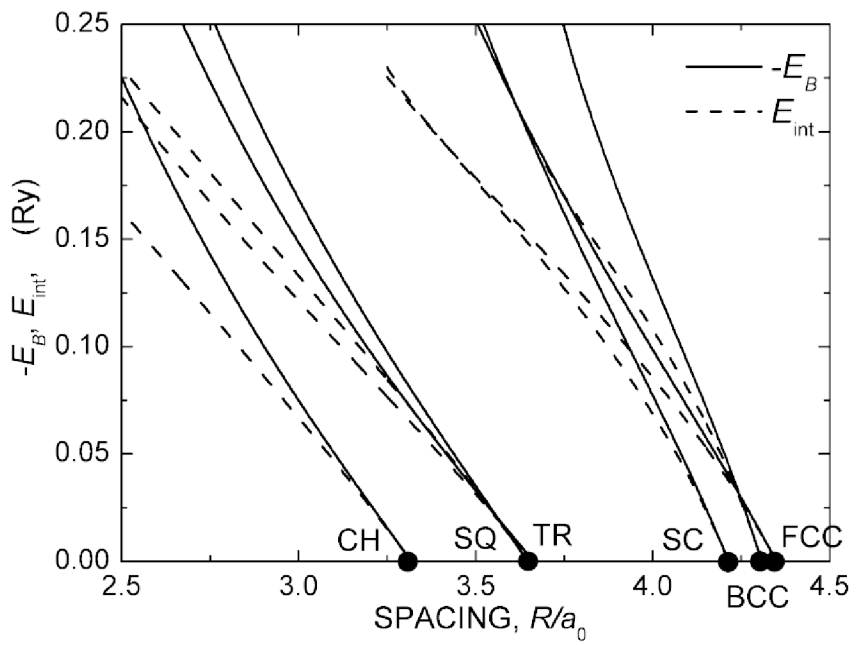

Fig. 3. The band $\left(-E_{\mathrm{B}}\right)$ and interaction $\left(E_{\text {int }}\right)$ energies near critical interatomic distance. The solid points mark the critical distances for MIT.

\section{Acknowledgments}

The authors acknowledge the grant No. 1 P03B 00129 from Ministry of Science and Higher Education.

\section{References}

[1] A. Rycerz, J. Spałek, Phys. Rev. B 65, 035110 (2002).

[2] A. Rycerz, Ph.D. Thesis, Uniwersytet Jagielloński, Kraków 2003; http://thwww.if.uj.edu.pl/ztms.

[3] E.M. Görlich, J. Kurzyk, A. Rycerz, R. Zahorbeński, R. Podsiadły, W. Wójcik, J. Spałek, in: Molecular Nanowires and Other Quantum Objects, Eds. A.S. Alexandrov, J. Demsar, I.Yanson, Kluwer Academic Publisher, Dordrecht 2004, p. 355 .

[4] J. Spałek, A. Rycerz, Phys. Rev. B 64, 161105 (2001); A. Rycerz, J. Spałek, Eur. Phys. J. B 40, 153 (2004).

[5] J. Spałek, E.M. Görlich, A. Rycerz, R. Zahorbeński, J. Phys., Condens. Matter 19, 25512 (2007); arXiv:cond-mat/0610815 (2006).

[6] M.C. Gutzwiller, Phys. Rev. Lett. 10, 159 (1963); Phys. Rev. 137, A1726 (1965).

[7] J. Kurzyk, J. Spałek, W. Wójcik, Acta Phys. Pol. A 111, 603 (2007); arXiv:0706.1266 (2007). 